Interactive multiobjective optimization for anatomy-based three-dimensional HDR brachytherapy

This article has been downloaded from IOPscience. Please scroll down to see the full text article. 2010 Phys. Med. Biol. 554703

(http://iopscience.iop.org/0031-9155/55/16/006)

View the table of contents for this issue, or go to the journal homepage for more

Download details:

IP Address: 130.237.41.141

The article was downloaded on 02/02/2012 at 13:47

Please note that terms and conditions apply. 


\title{
Interactive multiobjective optimization for anatomy-based three-dimensional HDR brachytherapy
}

\author{
Henri Ruotsalainen ${ }^{1}$, Kaisa Miettinen ${ }^{2}$, Jan-Erik Palmgren ${ }^{3}$ and \\ Tapani Lahtinen ${ }^{3}$ \\ ${ }^{1}$ Department of Physics and Mathematics, University of Eastern Finland, PO Box 1627, \\ FI-70211 Kuopio, Finland \\ ${ }^{2}$ Department of Mathematical Information Technology, PO Box 35 (Agora), FI-40014 University \\ of Jyväskylä, Finland \\ ${ }^{3}$ Department of Oncology, Kuopio University Hospital, PO Box 1777, FI-70211 Kuopio, Finland \\ E-mail: henrimatias.ruotsalainen@gmail.com
}

Received 22 April 2010, in final form 21 June 2010

Published 30 July 2010

Online at stacks.iop.org/PMB/55/4703

\begin{abstract}
In this paper, we present an anatomy-based three-dimensional dose optimization approach for HDR brachytherapy using interactive multiobjective optimization (IMOO). In brachytherapy, the goals are to irradiate a tumor without causing damage to healthy tissue. These goals are often conflicting, i.e. when one target is optimized the other will suffer, and the solution is a compromise between them. IMOO is capable of handling multiple and strongly conflicting objectives in a convenient way. With the IMOO approach, a treatment planner's knowledge is used to direct the optimization process. Thus, the weaknesses of widely used optimization techniques (e.g. defining weights, computational burden and trial-and-error planning) can be avoided, planning times can be shortened and the number of solutions to be calculated is small. Further, plan quality can be improved by finding advantageous trade-offs between the solutions. In addition, our approach offers an easy way to navigate among the obtained Pareto optimal solutions (i.e. different treatment plans). When considering a simulation model of clinical 3D HDR brachytherapy, the number of variables is significantly smaller compared to IMRT, for example. Thus, when solving the model, the CPU time is relatively short. This makes it possible to exploit IMOO to solve a 3D HDR brachytherapy optimization problem. To demonstrate the advantages of IMOO, two clinical examples of optimizing a gynecologic cervix cancer treatment plan are presented.
\end{abstract}

(Some figures in this article are in colour only in the electronic version) 


\section{Introduction}

Radiation delivery in high-dose-rate (HDR) intracavitary brachytherapy using an afterloading unit is realized by using temporarily implanted applicators: a programmable remote unit moves a single radioactive source along an applicator or applicators. This system produces a high-dose region centered on the planning target volume (PTV) while sparing the adjacent organs such as bladder and bowel. The flexibility of this system allows it to be tailored to a variety of different patient anatomy and cancer types because a wide variety of dose distributions can be generated from a given applicator simply by adjusting the length of time (dwell time) that the source dwells at any location within the applicator (dwell position). In clinics, this flexibility allows the full benefit of the use of a three-dimensional (3D) planning system based on computer tomography (CT) or magnetic resonance imaging (MRI).

However, the increased flexibility in treatment applications and imaging also increases the complexity in the treatment planning. A patient domain can be divided into three different parts based on a patient anatomy: a PTV, dose-sensitive organs at risk (OARs) and healthy normal tissue (NT). The OARs and NT are typically near the PTV, and thus, they may be unnecessarily overdosed. To maintain a complete coverage of the PTV and simultaneously reduce the dose to NT and OARs, the dose distribution should be as conformal as possible to the relevant anatomy and, thus, treatment plans must be carefully constructed.

The aim of brachytherapy is to treat the tumor without affecting healthy tissue but, naturally, increasing the dose in the tumor also increases the unwanted dose in the surrounding healthy tissue. Thus, when one target is optimized, the other will suffer, and the solution is a compromise between them. This trade-off is complex, and optimization tools capable of handling multiple and conflicting objectives are naturally required. Besides inverse planning (Lessard and Pouliot 2001, Lessard et al 2002, Hsu et al 2004, Pouliot et al 2004, DeWitt et al 2005, Yasuo et al 2005, Sumida et al 2006, Alterovitz et al 2006, Chajon et al 2007, Morton et al 2008), there has been interest in using multiobjective optimization in brachytherapy treatment planning (e.g. Yu 1997, Lahanas et al 1999, Yu et al 2000, Lessard et al 2006). The multiobjective optimization approaches presented in the literature are based on using objective weights defined beforehand, where the final objective function is expressed as a weighted sum of the conflicting objectives (e.g. Milickovic et al 2002, Lahanas and Baltas 2003). In these cases, objectives are often formulated as using penalties where doses exceeding predefined upper limits are penalized (e.g. Lahanas et al 2003b), and the approach is the same often in inverse planning, too. Unfortunately, it is typically hard to predefine the priorities or weights of the optimization targets. Moreover, sometimes information about objectives and even the practical relevance of the objective functions can become blurred if the objectives are expressed as a sum. Furthermore, penalizing only the overdose should not be the actual goal as we suggest in this paper. Alternatively, evolutionary algorithms (e.g. Lahanas et al 2001, Milickovic et al 2001) have been used, too. These methods have their own difficulties because they are time consuming requiring a lot of calculation when computing a large set of approximating solutions. Similar optimization methods and methods how to compare different solutions have been studied also in intensity modulated radiotherapy (IMRT) treatment planning in Küfer et al (2003, 2005), Meyer et al (2004), Craft et al (2005, 2007), Halabi et al (2006), Hoffmann et al (2006), Thieke et al (2007), Ehrgott and Winz (2008), Monz et al (2008), Craft and Bortfeld (2008). However, the optimization methods used are not interactive (as interactivity is defined in Miettinen (1999)) or those are based on beforehand calculated database of solutions from where the final solution is chosen. Still, it is not well known how to navigate around the solutions in order to find the final solution. 


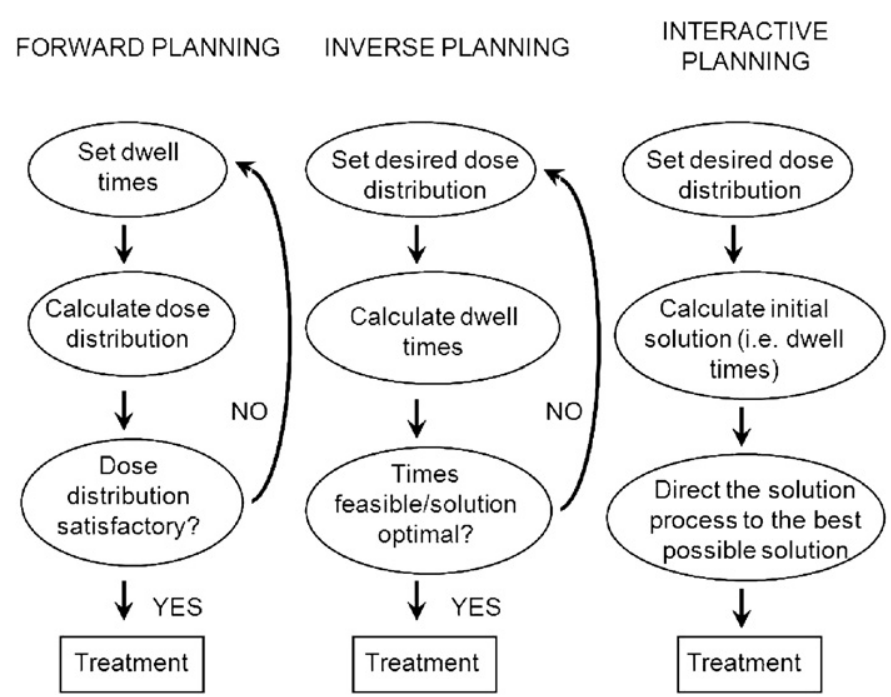

Figure 1. Philosophies underpinning forward planning, inverse planning and interactive planning (see also Ehrgott and Winz (2008)).

To overcome some shortcomings of currently used approaches, we exploit an interactive multiobjective optimization (IMOO) method for 3D HDR brachytherapy optimization in this paper. By interactivity we mean that the treatment planner directs the search for most preferred solution with preference information that is understandable and intuitive for her $/ \mathrm{him}$, and Pareto optimal solutions are based on this preference information. Thus, the treatment planner is in control and only solutions interesting to her/him are computed. For some reason, IMOO methods have not been studied in the field of brachytherapy optimization before. As mentioned, the studies where brachytherapy treatment plans have been optimized are based on a priori methods (weighting method, e.g. Milickovic et al 2002) or a posteriori methods (evolutionary algorithms, e.g. Lahanas et al 2003b). The biggest difference between the approaches is that in our approach the treatment planner's preferences are used during the iterative optimization process to direct the search in order to find the most preferred plan (the best Pareto optimal solution) between the conflicting treatment planning targets. This interactivity can be carried out in clinical 3D patient cases since the number of variables and the size of the problem is relative small (e.g. compared to IMRT instances). IMOO makes treatment planning times shorter (because there is no need for computing a database of Pareto optimal solutions beforehand which can take 2-3 h, for example Thieke et al (2007)), and good trade-offs between the targets can be found to improve the treatment plan's quality. Furthermore, the interactive approach improves the treatment planner's control over treatment: with this system, the treatment planner plays directly with the compromises between target coverage and protection of OARs instead of with dwell positions, dwell times and objective weights as shown in figure 1.

Because of this reasoning, according to our experience, IMOO suits well for brachytherapy optimization, and we demonstrate the advantages of our approach by examples of a treatment plan of cervical cancer. In these examples, the real multiobjective nature of the problem is taken into account in the problem formulation and in the interactive solution process. We want to point out that there is not much space for improvement of the final treatment plan but the strength of our approach is its interactivity. Our approach brings the planning process 
near to the real clinical issues avoiding artificial simplifications, and when compared to the currently used trial-and-error method, our approach guarantees the (Pareto) optimality of the final solution, i.e. treatment plan. Let us emphasize that these kinds of tools are designed to assist human treatment planners in their work, not to replace them.

\section{Methods}

\subsection{Dose calculation}

Before optimization, the dose distribution in a patient needs to be calculated. The dose $D(x)$ at the $i$ th sampling (dose calculation) point $x$ is calculated by

$$
D_{i}(x)=\sum_{j=1}^{p} t_{j} d_{i j},
$$

where $p$ is the number of source dwell positions, $t_{j}$ is the dwell time of the $j$ th source dwell position and $d_{i j}$ is the kernel value, i.e. dose value, for the $i$ th sampling point and $j$ th source dwell position. The dose rate matrix $d_{i j}$ can be calculated using the following equation according to TG43 (Nath et al 1995, Rivard et al 2004):

$$
d_{i j}=S_{k} \Lambda \Phi_{a n}\left(\theta, r_{i j}\right) g\left(r_{i j}\right) / r_{i j}^{2},
$$

where $S_{k}$ is the air kerma strength, $\Lambda$ is the dose-rate constant, $\Phi_{a n}\left(\theta, r_{i j}\right)$ is the anisotropy function, $g\left(r_{i j}\right)$ is the radial dose functions and $r_{i j}$ is the distance between the source dwell position $j$ (point source) and the sampling point $i$. In this paper, we use equation (1) for dose calculations with a simplification made to the kernel $d_{i j}$ : the source anisotropy is neglected, i.e. $\Phi_{a n}\left(\theta, r_{i j}\right)=1$. In IMOO, the dwell times $t_{j}$ (let us denote $t_{j}=t$ in the future) are the decision variables and the sampling points are selected in the neighborhood of the PTV. Thus, the problem size is substantially smaller than in IMRT, for example.

\subsection{Objective function formulation}

The aim of brachytherapy treatment planning is to obtain a plan which covers the PTV with at least some specified dose value $D_{\mathrm{PTV}}$, which is case-specific depending on the type of the tumor. In addition to this, there is an upper bound (ESTRO's guideline (Haie-Meder et al 2005, Pötter et al 2006)) for the dose in NT and the OAR which should not be exceeded. We denote these bounds by $D_{\mathrm{NT}}$ and $D_{\mathrm{OAR}}$, respectively. Traditionally in optimization, dwell times $t$ are sought so that the above-mentioned requirements are fulfilled, that is

$$
\begin{array}{ll}
D(x) \geqslant D_{\mathrm{PTV}}, & x \in \mathrm{PTV}, \\
D(x) \leqslant D_{\mathrm{NT}}, & x \in \mathrm{NT}, \\
D(x) \leqslant D_{\mathrm{OAR}}, & x \in \mathrm{OAR} .
\end{array}
$$

In the literature, several objective functions have been used to fulfill these requirements: variance-based objective functions (e.g. Lahanas et al 2003a) or dose volume histogram (DVH)-based objective functions (e.g. Lahanas et al 2003b), for example. In addition, the formulation used in Lessard and Pouliot (2001) and Lessard et al (2002) is well known. However, let us point out that even though (3) describes an acceptable solution, it is important to carefully think what should actually be optimized: the goal is that the dose in NT and OAR should be as low as possible (minimized), not only under the predefined bounds $D_{\mathrm{NT}}$ and $D_{\text {OAR, }}$ respectively.

Now, based on the fact that we want to minimize the dose in NT and OAR, objective functions can be formulated (in a discrete form) as 


$$
\begin{aligned}
f_{1}(t) & =\frac{\tilde{I}_{\mathrm{PTV}}}{I_{\mathrm{PTV}}}, \\
f_{2}(t) & =\frac{1}{I_{\mathrm{NT}}} \sum_{x \in \mathrm{NT}} D(x), \\
f_{3}(t) & =\frac{1}{I_{\mathrm{OAR}}} \sum_{x \in \mathrm{OAR}} D(x), \\
f_{4}(t) & =\max _{x \in \mathrm{PTV}} D(x),
\end{aligned}
$$

and

$$
f_{5}(t)=\max _{x \in \mathrm{PTV}}\left|D_{\mathrm{PTV}}-D(x)\right|,
$$

where $I_{\mathrm{PTV}}, I_{\mathrm{NT}}$ and $I_{\mathrm{OAR}}$ denote numbers of sampling points in a region (PTV, NT and OAR). Because of computational reasons, all the sampling points are situated on the surface of the region in question. Thus, a maximum dose inside the PTV is not an objective but it is controlled later. Here, $\tilde{I}_{\mathrm{PTV}}$ represents the number of sampling points on the PTV that have a dose value bigger than the dose limit $D_{\mathrm{PTV}}$. Thus, the function $f_{1}$ represents a percentage volume where the dose is higher or equally high to the prescribed dose $D_{\mathrm{PTV}}$ on the PTV, and it is maximized. The functions $f_{2}$ and $f_{3}$ are the averaged doses on NT and the OAR, respectively, to be minimized. If there are multiple OARs (as also here in the examples), there are as many objective functions each similar to $f_{3}$. The objective function $f_{4}$ describes the maximum dose on the PTV surface. The objective $f_{5}$ represents the maximum dose deviation from a desired dose $D_{\mathrm{PTV}}$ on the PTV (to be minimized). Therefore, both objective functions $f_{1}$ and $f_{5}$ can be individually used to control the dose on the PTV. With these objective functions, the unwanted dose on NT and the OAR is really minimized, not only penalized if it exceeds predefined upper limits for doses.

The objective function $f_{1}$ is non-convex (which makes the optimization problem also nonconvex) (Deasy 1997) whereas $f_{5}$ is convex (and the optimization problem is also convex). There are several ways to convert non-convex objectives into convex ones (Romeijn et al 2004), and this feature has been utilized in IMRT optimization (Romeijn et al 2003, Craft et al 2005, 2007, Halabi et al 2006). The reasons to modify objective functions into convex form are clear: many optimization approaches are capable of solving convex optimization problems quickly and exactly, and non-convex objectives cause problems in finding all Pareto optimal solutions when using objective weights (Miettinen 1999, Romeijn et al 2004). Besides, they necessitate the use of global optimizers. However, here we use an optimization method capable of solving non-convex multiobjective optimization problems. Thus, it is not necessary to use convex functions in optimization. For comparison, we solve convex and non-convex optimization problems in the examples. Let us mention that the optimization method we are using is able to find any Pareto optimal solutions since there are no objective weights used.

\subsection{Multiobjective optimization}

2.3.1. Multiobjective optimization problem. A multiobjective optimization problem can be defined as follows (Miettinen 1999):

$$
\begin{array}{ll}
\text { minimize } & \left\{f_{1}(t), f_{2}(t), \ldots, f_{k}(t)\right\} \\
\text { subject to } & t \in S,
\end{array}
$$

where $t$ is a vector of decision variables from the feasible set $S \subset \boldsymbol{R}^{n}$ defined by linear, nonlinear and box constraints. We can denote an objective vector by $f(t)=$ 
$\left(f_{1}(t), f_{2}(t), \ldots, f_{k}(t)\right)^{T}$. Furthermore, we denote the image of the feasible set by $\boldsymbol{f}(S)=\mathrm{Z}$ and call it a feasible objective set.

In multiobjective optimization, optimality is understood in the sense of Pareto optimality (Miettinen 1999). All the Pareto optimal solutions are equally good from a mathematical point of view, and they can be regarded as equally valid compromise solutions of the problem. There exists no trivial mathematical tool in order to find the best solution in the Pareto optimal set because vectors cannot be ordered completely. That is why we need some additional information. Typically, a decision maker (here, a treatment planner), who is an expert in the field, is needed in order to find the best or most satisfying solution, called the final one. The decision maker can participate in the solution process, and, in one way or another, determine which of the Pareto optimal solutions is the most satisfying to be the final solution.

According to Miettinen (1999), the methods developed for multiobjective optimization can be divided into four classes depending on the role of the decision maker. There are methods for use when no decision maker is available. In these methods, the final solution is some neutral compromise solution. In the three other classes, the decision maker participates in the solution process beforehand, afterward or iteratively: these methods are called a priori, a posteriori and interactive methods, respectively. It can be difficult for the decision maker to specify preferences (e.g. objective weights) before the solution process has started and, on the other hand, generating many Pareto optimal solutions (e.g. at least 75 solutions (Craft and Bortfeld 2008) and more if using evolutionary algorithms) for the decision maker to compare can be computationally costly. It is also problematic to compare many solutions without setting too much cognitive load on the decision maker. Consequently, and encouraged by experiences reported in Ruotsalainen et al (2009), in this paper we concentrate on interactive methods.

2.3.2. The interactive multiobjective optimization method. NIMBUS In this paper, we integrate an anatomy-based 3D HDR brachytherapy dose calculation model with an IMOO method. The method we use is NIMBUS (Miettinen and Mäkelä 1995, Miettinen 1999, Miettinen and Mäkelä 2006). This method has been successfully used in external radiotherapy treatment planning optimization in a 2D academic case (Ruotsalainen et al 2009).

In IMOO methods, the information given to and required from the decision maker must be easily understandable. The NIMBUS method is based on the idea of classification of objective functions. It is known that classification can be considered an acceptable task for human decision makers from a cognitive point of view (Larichev 1992). In NIMBUS, the decision maker participates in the solution process iteratively and continuously. Finally, he/she decides which of the Pareto optimal solutions obtained is the most desired one. During the solution process, the decision maker classifies objective functions at the current Pareto optimal point into up to five classes. The classes are as follows:

$-I^{\text {imp }}$ functions whose values should be improved,

$-I^{\text {asp }}$ functions whose values should be improved until to a desired aspiration level,

$-I^{\text {sat }}$ functions whose values are satisfactory,

$-I^{\text {bound }}$ functions whose values can be allowed to worsen until they reach a given bound,

$-I^{\text {free }}$ functions whose values can change freely.

Since all the solutions considered are Pareto optimal, the decision maker cannot make a classification where all the objective function values should improve without allowing at least one of the objective functions to be impaired. The aspiration levels and the 
bounds are elicited from the decision maker during the classification procedure if they are needed.

By classifying the objective functions, the decision maker gives preference information about how the current solution should be improved. Based on that, a scalarized single objective optimization problem (a subproblem, as we call it) can be formed, and it can be solved with an appropriate optimizer. Here, we use a synchronous NIMBUS method (Miettinen and Mäkelä 2006). In this method, there are four different subproblems available, so the decision maker can choose whether to see one to four new solutions after each classification. Each subproblem generates a new Pareto optimal solution that satisfies the preferences given in the classification as much as possible, but the preferences are taken into account in slightly different ways (Miettinen and Mäkelä 2002). The decision maker can use any solution obtained so far as a starting point for a new classification. Interesting solutions can also be saved in a database, so that the solution process can be continued later from any of them. Alternatively, the decision maker can generate a desired number of Pareto optimal intermediate solutions between any two solutions. This differs from many other approaches used in treatment planning in which intermediate solutions are only approximated, see e.g. Monz et al (2008). For more information about the NIMBUS algorithm, the scalarizations used, generation of intermediate solutions and ways of aiding comparison of Pareto optimal solutions generated with different visualizations, see Miettinen and Mäkelä (2006).

\section{Results}

\subsection{Problem settings}

Here, two clinical examples of seeking dwell time values of a source in a gynecologic cervix cancer treatment are presented. In the examples, Fletcher-Suit and Ring intracavitary applicators (manufactured by Nucletron) were used to deliver the radiation. In the first example, there were 17 possible dwell positions (resolution of $5 \mathrm{~mm}$ in three applicators, Fletcher-Suit intracavitary applicator system). Thus, the number of continuous decision variables was 17 . In addition, the number of sampling points was 508. In the second example, the number of possible dwell positions (decision variables) was 21 (resolution of $5 \mathrm{~mm}$ in two applicators, ring intracavitary applicator system), and the number of sampling points was 473. Thus, the problem size is small compared to a 3D IMRT case which can easily have over 10000 continuous decision variables. The problems contained box constraints for the decision variables (i.e. dwell times). In the first example, there were two OARs (bladder and rectum; sigmoid (also called sigma) was not adjacent to the tumor), and thus, there were two objective functions similar to $f_{3}\left(f_{3}^{\text {bladder }}\right.$ and $\left.f_{3}^{\text {rectum }}\right)$. In the second example, there were three OARs (bladder, rectum and sigmoid) and thus three objective functions similar to $f_{3}\left(f_{3}^{\text {bladder }}\right.$, $f_{3}^{\text {rectum }}$ and $f_{3}^{\text {sigmoid }}$ ).

In these examples, all the simulations were carried out with the mathematical software Matlab ${ }^{\circledR}$ R2006b after the patient geometries (anatomy, dwell positions and sampling points) were generated with a treatment planning software (BrachyVision ${ }^{\circledR}$, Varian Medical Systems, software version 7.3.10) at the Kuopio University Hospital. The optimization was done with a personal computer (Pentium ${ }^{\circledR} 4 \mathrm{CPU} 3.00 \mathrm{GHz}$ with $2 \mathrm{~GB}$ central memory). For IMOO, an implementation of the NIMBUS method, called IND-NIMBUS ${ }^{\circledR}$, was used (Miettinen 2006). In the first example, a global optimization method (computation time was minutes per classification with the presented PC) was used to solve the formed subproblems because the optimization problem is non-convex. The faster (computation time was only a few seconds 
per classification) local optimization method was used in the second example (since the optimization problem is convex, a global method is not needed).

\subsection{Example 1: Fletcher-Suit applicator}

The first optimization problem used for the demonstration of the proposed IMOO approach has the form

$$
\begin{array}{ll}
\text { optimize } & \left\{f_{1}(t), f_{2}(t), f_{3}^{\text {bladder }}(t), f_{3}^{\text {rectum }}(t), f_{4}(t)\right\} \\
\text { subject to } & t \in S,
\end{array}
$$

where $t$ is a vector of continuous decision variables, and $S=[0,100 \mathrm{~s}] \times[0,100 \mathrm{~s}]$ $\times \cdots \times[0,100 \mathrm{~s}] \subset \boldsymbol{R}^{17}$. The objective functions $f_{1}-f_{4}$ we defined in section $2.2: f_{1}$ represents the percentage value of sampling points in the PTV which has a dose value higher than the dose limit $D_{\text {PTV }}(7 \mathrm{~Gy})$, and $f_{2}, f_{3}^{\text {bladder }}, f_{3}^{\text {rectum }}$ represent averaged doses in NT, bladder and rectum, respectively (in Gy). Finally, $f_{4}$ is the maximum dose on the PTV surface (in Gy).

3.2.1. Interactive solution process. In this optimization problem, the interactive solution process (i.e. moving from one Pareto optimal solution to another) was guided by preference information of a treatment planner, who was acting as a decision maker. Before the solution process, the treatment planner had the following desires: the percentage value of sampling points on the surface of PTV that have a dose value higher than the dose limit $D_{\text {PTV }}$ should be maximized $\left(f_{1}\right)$. At the same time, the averaged doses on NT and both OARs should be minimized $\left(f_{2}, f_{3}^{\text {bladder }}, f_{3}^{\text {rectum }}\right)$. Since the objective function $f_{4}$ shows the maximum dose on the PTV surface, he wanted to minimize it, too. At the very beginning, the objective functions had the initial values (generated by IND-NIMBUS) $f_{1}=0.58, f_{2}=9.89, f_{3}^{\text {bladder }}=1.96, f_{3}^{\text {rectum }}=3.13$ and $f_{4}=96.80$ (initial solution $\boldsymbol{f}\left(t^{1}\right)$ ). As can be seen from the initial objective function values $\boldsymbol{f}\left(t^{1}\right)$, the $f_{1}$ value was certainly too low ( $f_{1}=0.58$, i.e. $58 \%$ of the PTV received higher dose than $D_{\text {PTV }}$ which was 7 Gy). Nevertheless, the objective functions $f_{2}, f_{3}^{\text {bladder }}$ and $f_{3}^{\text {rectum }}$ were in a good level and, thus, the dose on NT and the OARs was low. However, the dose coverage of the PTV was too low and the tumor would not be treated properly. Thus, the treatment planner wanted to search for a better solution in an iterative way. He started to classify the functions and generated new solutions (see classes in section 2.3.2), and in this way declared his preferences and steered the solution process interactively and iteratively toward the most satisfying Pareto optimal solutions.

In the first classification, as said, he wanted to obtain a better value of $f_{1}$ (aspiration level 0.70 ) and simultaneously maintain the good values of $f_{3}$ (save the OARs). Thus, he set a bound to $f_{3}^{\text {bladder }}$, and an aspiration level to $f_{3}^{\text {rectum }}$. The bound and aspiration level both were 2.00. At the same time, he had to allow some other objectives $\left(f_{2}\right.$ and $\left.f_{4}\right)$ to get worse. That is, the classification was $f_{1}: I^{\text {asp } 0.70}, f_{2}: I^{\text {free }}, f_{3}^{\text {bladder }}: I^{\text {bound } 2.00}, f_{3}^{\text {rectum }}: I^{\text {asp } 2.00}$ and $f_{4}: I^{\text {free }}$, and he wanted to generate one new solution (solutions are collected in table 1). After the first classification, the treatment planner obtained a better solution $\left(f\left(t^{2}\right)\right)$ because the rectum $\left(f_{3}^{\text {rectum }}=2.74\right)$, which he considered very important, obtained a lower dose than in the solution $\boldsymbol{f}\left(t^{1}\right)$, but still $f_{1}$ was too low according to his preferences. Because of this reason, he decided to do a second classification using the solution $f\left(t^{2}\right)$ as a starting point. In this classification, the treatment planner wanted to improve $f_{1}$ as much as possible, and again, he set a bound to $f_{3}^{\text {bladder }}$, and an aspiration level to $f_{3}^{\text {rectum }}$ to maintain the good levels of these objectives. In addition, he allowed $f_{2}$ and $f_{4}$ to change freely as before. 


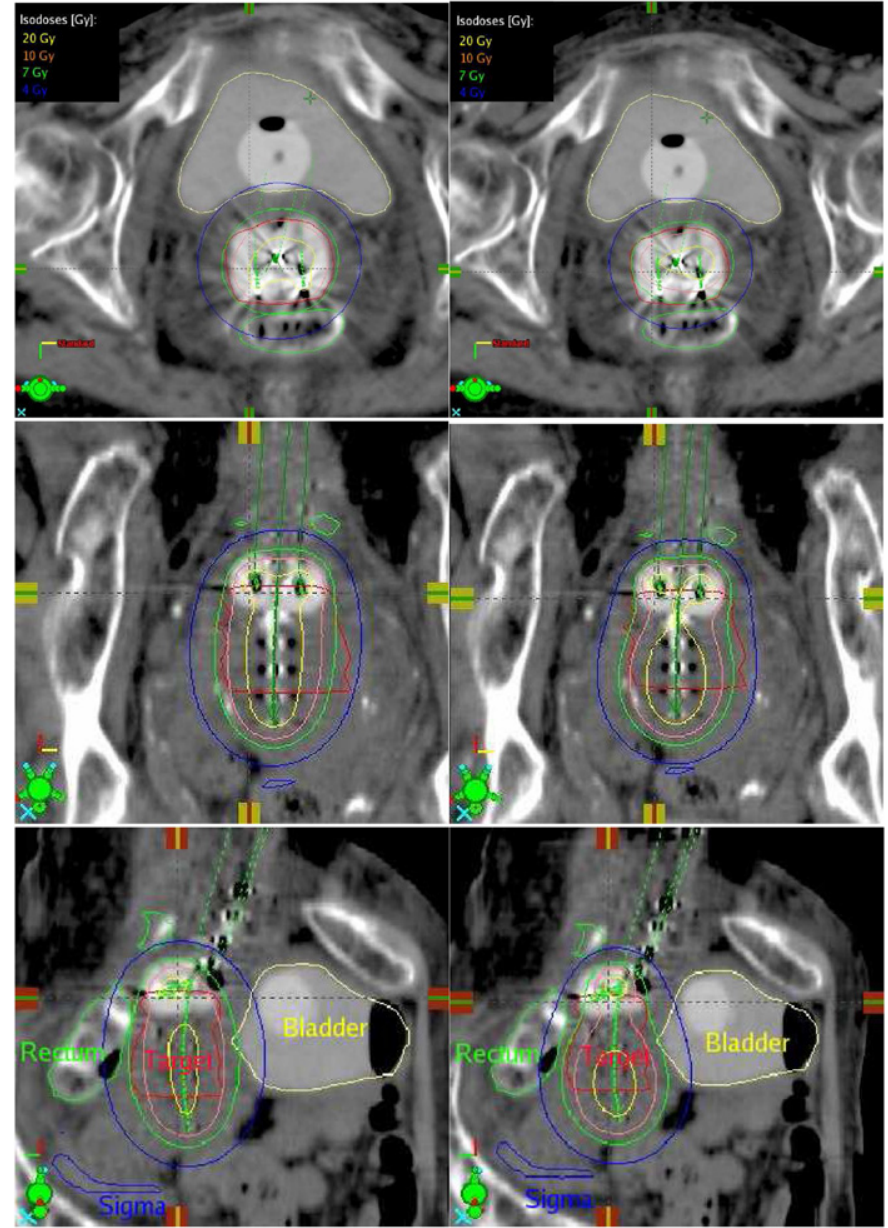

Figure 2. Example 1: left, the final dose distribution with IMOO (solution $\boldsymbol{f}\left(t^{9}\right)$ ) from different points of view ( $x$-, $y$ - and $z$-directions), and right, for comparison, solution obtained with BrachyVision ${ }^{\circledR}$ optimization. Isodose of $7 \mathrm{~Gy}$ (prescribed dose in target) is the second outermost isodose curve (green in the electronic version). In the electronic version only, target is red, rectum is green, bladder is yellow and sigmoid is blue.

Therefore, the classification was $f_{1}: I^{\text {imp }}, f_{2}: I^{\text {free }}, f_{3}^{\text {bladder }}: I^{\text {bound 2.00 }}, f_{3}^{\text {rectum }}: I^{\text {asp } 2.00}$ and $f_{4}: I^{\text {free }}$. After the second classification, the treatment planner obtained three new solutions $\boldsymbol{f}\left(t^{3}\right)-\boldsymbol{f}\left(t^{5}\right)$ having excellent $f_{1}$ values, but, at the same time, values of other objectives were not so good (table 1). That is why he wanted to generate five intermediate solutions between

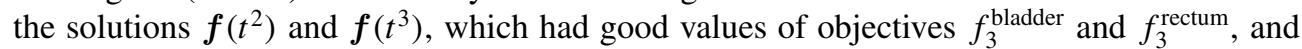
$f_{1}$, respectively. Intermediate solutions $\boldsymbol{f}\left(t^{6}\right)-\boldsymbol{f}\left(t^{10}\right)$ represent Pareto optimal solutions between the conflicting treatment planning targets, and now the treatment planner was able to choose the best Pareto optimal solution according to his knowledge to be the final solution, i.e. the final treatment plan. That solution was $f\left(t^{9}\right)$, in which the objective values were $f_{1}=0.73, f_{2}=10.50, f_{3}^{\text {bladder }}=2.35, f_{3}^{\text {rectum }}=3.15$ and $f_{4}=52.23$. As can be seen in figures 2 (left) and 3, all the requirements of the treatment plan were taken into account as much as possible: harmful dose in rectum and bladder was minimized and the prescribed dose in the PTV was delivered. Thus, the treatment plan was clinically acceptable. 


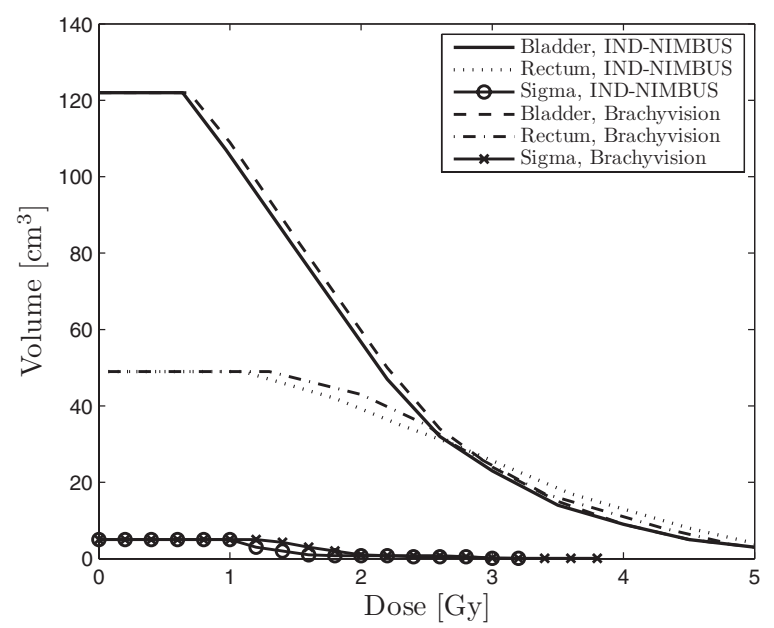

Figure 3. Example 1: for comparison, DVHs of OARs for two solutions. DVH values start to decrease faster in the IMOO solution than in the solution obtained with BrachyVision ${ }^{\circledR}$.

Table 1. Summary of the IMOO solution process for example 1. Bounds and aspiration levels used are denoted as superscripts in the classification notation. Ideal and nadir represent approximated ranges of objective function values in Pareto optimal solutions.

\begin{tabular}{|c|c|c|c|c|c|}
\hline Solution & $f_{1}(-)$ & $f_{2}(\mathrm{~Gy})$ & $f_{3}^{\text {bladder }}(\mathrm{Gy})$ & $f_{3}^{\text {rectum }}(\mathrm{Gy})$ & $f_{4}(\mathrm{~Gy})$ \\
\hline Ideal & 1 & 0 & 0 & 0 & 0 \\
\hline Nadir & 0 & 25.82 & 4.64 & 7.72 & 744.33 \\
\hline \multicolumn{6}{|l|}{ Initial solution } \\
\hline $\boldsymbol{f}\left(t^{1}\right)$ & 0.58 & 9.89 & 1.96 & 3.13 & 96.80 \\
\hline First classification & $I^{\text {asp } 0.70}$ & $I^{\text {free }}$ & $I^{\text {bound } 2.00}$ & $I^{\operatorname{asp} 2.00}$ & $I^{\text {free }}$ \\
\hline $\boldsymbol{f}\left(t^{2}\right)$ & 0.58 & 9.55 & 1.98 & 2.74 & 135.89 \\
\hline Second classification & $I^{\mathrm{imp}}$ & $I^{\text {free }}$ & $I^{\text {bound } 2.00}$ & $I^{\text {asp } 2.00}$ & $I^{\text {free }}$ \\
\hline $\boldsymbol{f}\left(t^{3}\right)$ & 0.83 & 11.18 & 2.67 & 3.29 & 55.18 \\
\hline $\boldsymbol{f}\left(t^{4}\right)$ & 1.00 & 15.86 & 3.34 & 4.05 & 109.89 \\
\hline $\boldsymbol{f}\left(t^{5}\right)$ & 0.79 & 11.57 & 2.55 & 3.24 & 76.85 \\
\hline \multicolumn{6}{|l|}{ Intermediate solutions ${ }^{\mathrm{a}}$} \\
\hline $\boldsymbol{f}\left(t^{6}\right)$ & 0.64 & 9.87 & 2.12 & 2.86 & 64.23 \\
\hline $\boldsymbol{f}\left(t^{7}\right)$ & 0.69 & 10.02 & 2.24 & 2.90 & 62.11 \\
\hline $\boldsymbol{f}\left(t^{8}\right)$ & 0.71 & 10.44 & 2.36 & 3.04 & 52.23 \\
\hline $\boldsymbol{f}\left(t^{9}\right)$ & 0.73 & 10.50 & 2.35 & 3.15 & 52.23 \\
\hline $\boldsymbol{f}\left(t^{10}\right)$ & 0.76 & 10.87 & 2.45 & 3.22 & 54.00 \\
\hline
\end{tabular}

${ }^{\text {a }}$ Intermediate solutions between $\boldsymbol{f}\left(t^{2}\right)$ and $\boldsymbol{f}\left(t^{3}\right)$.

All the solutions obtained and steps taken by the treatment planner during the solution process are collected in table 1 . In this table, the starting point of a new classification and the final solution are given in bold face. Let us add that a more thorough description of a typical process of classifying objective functions in a radiotherapy case and steering the optimization process is presented in Ruotsalainen et al (2009). 


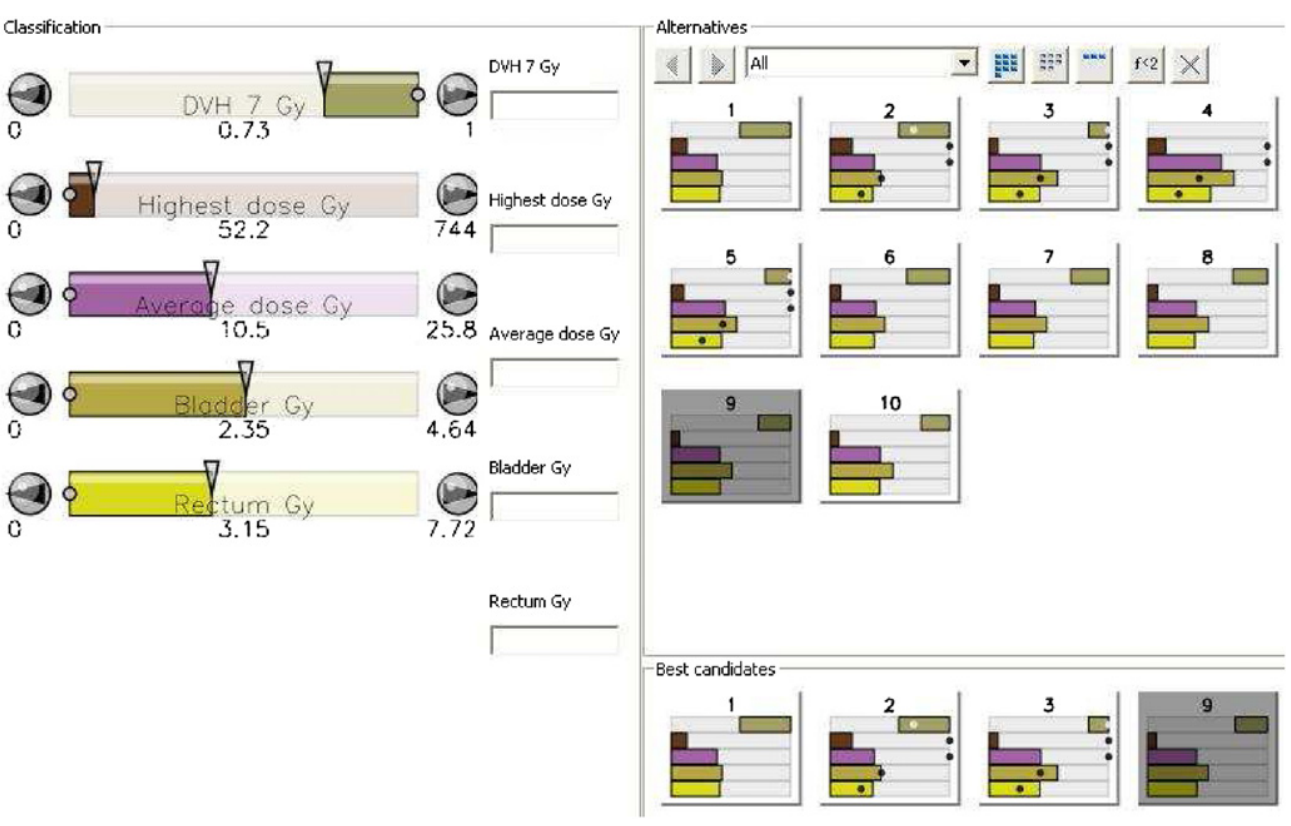

Figure 4. Example 1: all the solutions obtained during the optimization process can be compared with a graphical tool in IND-NIMBUS. On the left, the final solution $\boldsymbol{f}\left(t^{9}\right)$ presented with bars describing objective function values, and, on the right, all ten solutions generated. On the lower right, best candidates, that is, solutions used in classifications and generating intermediate solutions. Solutions can be visualized also with value path, whisker plot, petal diagram or spider web, for example Miettinen and Mäkelä (2006).

3.2.2. Comparison and discussion. In this example, we have shown how our IMOO approach can handle the strongly conflicting objectives in a cervix cancer case. As can be seen in figure 4 (a display of IND-NIMBUS software), the solutions obtained can be compared and carefully studied during the interactive solution process. Thus, the treatment planner (decision maker) is better prepared to make the final decision, i.e. choose the final treatment plan, after analyzing the isodose maps (figure 2), DVHs (figure 3), graphical information (figure 4) and numerical information (table 1 and figure 4). Similar ideas for presenting information for decision makers have been presented also in Küfer et al (2003), Thieke et al (2007), Craft et al (2007), Monz et al (2008), Ehrgott and Winz (2008), for example. Compared to the trial-and-error method of finding appropriate weights or methods demanding a large database of Pareto optimal solutions, our approach makes treatment planning times shorter, and a good trade-off between the objectives can be found to improve the treatment plan's quality. A good example of this trade-off information can be seen in table 1: when comparing solutions $f\left(t^{1}\right)$ and $f\left(t^{2}\right)$, the dose in the rectum could be decreased without losing the target coverage, for example.

For comparison, in figures 2 (right) and 3, a treatment plan obtained with the BrachyVision ${ }^{\circledR}$ optimization tool is presented. In the BrachyVision ${ }^{\circledR}$ optimization tool, the objectives are formulated as weighted penalties penalizing doses exceeding the following limits: bladder $6.2 \mathrm{~Gy}$, rectum $4.9 \mathrm{~Gy}$ and sigmoid $4.5 \mathrm{~Gy}$. In addition, dose in the PTV should be at least 7.2 Gy. These limits are based on ESTRO's guideline (Haie-Meder et al 2005, Pötter et al 2006). The same treatment planner also executed the treatment planning 
Table 2. Example 1: comparison of two solutions with PTV $90 \%$ values, OAR $2 \mathrm{~cm}^{3}$ values and volumes of NT exceeding $50 \%$ of $D_{\mathrm{PTV}}$.

\begin{tabular}{lll}
\hline & IMOO (Gy) & BrachyVision $^{\circledR}(\mathrm{Gy})$ \\
\hline PTV 90\% & 7.32 & 7.23 \\
Bladder 2 cm & 5.24 & 5.06 \\
Rectum $2 \mathrm{~cm}^{3}$ & 5.64 & 5.01 \\
Sigmoid 2 cm & 1.38 & 1.61 \\
NT 3.5 Gy & $177.9 \mathrm{~cm}^{3}$ & $200.4 \mathrm{~cm}^{3}$ \\
\hline
\end{tabular}

optimization using the BrachyVision ${ }^{\circledR}$ optimization tool. The time required for this procedure was about half an hour (IND-NIMBUS took about $20 \mathrm{~min}$ ) because the treatment planner had to vary the weighting factors many times, and he was not able to say how they should be changed. As stated, the weighting factors are not intuitive and thus it took such a long time to obtain an acceptable solution. For that reason, the BrachyVision ${ }^{\circledR}$ optimization is not in use in this hospital but the treatment planner prefers the trial-and-error manipulation of dwell times and positions for treatment planning.

When comparing the results, it can be seen in figure 3 that DVH values start to decline clearly faster in the IMOO solution than in the BrachyVision ${ }^{\circledR}$ solution, which is good. Table 2 presents PTV 90\% dose volume values, OAR $2 \mathrm{~cm}^{3}$ dose volume values and NT volumes where $50 \%$ of $D_{\text {PTV }}$ is exceeded for the two solutions. Here, NT also includes OARs but not the PTV. Threshold value $50 \%$ of $D_{\text {PTV }}$ was selected because that dose can cause secondary effects to NT. When comparing the values in table 2, we can see that PTV is covered with radiation better and the doses in sigmoid and NT are smaller in the solution obtained with IMOO. When PTV exceeds the prescribed dose (here, 7 Gy) it is not generally true that a higher value is better (especially not in an IMRT case). However, in brachytherapy in which a radiation source is located inside a patient (in PTV) it is not that critical, and high dose can be allowed in order to obtain as conformal a dose distribution as possible to the PTV. In this case, the better dose coverage of the PTV can be seen from isodose maps. Since there are conflicting targets and trading-off occurs between them, we can observe that the dose in bladder and rectum is smaller in the solution obtained with BrachyVision ${ }^{\circledR}$. However, the DVH reveals (in figure 3 ) that the IMOO solution as a whole is better than the BrachyVision ${ }^{\circledR}$ solution.

\subsection{Example 2: ring applicator}

In example 2, the optimization problem has the form

$$
\begin{array}{ll}
\text { optimize } & \left\{f_{2}(t), f_{3}^{\text {bladder }}(t), f_{3}^{\text {rectum }}(t), f_{3}^{\text {sigmoid }}(t), f_{4}(t), f_{5}(t)\right\} \\
\text { subject to } & t \in S .
\end{array}
$$

The functions $f_{2}-f_{4}$ are the same as in the previous example but $f_{5}$ represents the maximum dose deviation from a desired dose $D_{\text {PTV }}(7 \mathrm{~Gy}$ ) on the PTV (to be minimized). Thus, the dose on the PTV is now controlled with $f_{5}$, not with $f_{1}$ (percentage volume where the dose is higher or equally high to $D_{\mathrm{PTV}}$ ) as in the previous example.

The treatment planner solved the problem in an interactive way as in example 1, and the final solution (obtained after four classifications which took in all about $10 \mathrm{~min}$ ) had objective function values $f_{2}=6.70, f_{3}^{\text {bladder }}=1.68, f_{3}^{\text {rectum }}=1.64, f_{3}^{\text {sigmoid }}=1.87, f_{4}=18.83$ and $f_{5}=2.88$ (in Gy). Here, for saving space, the results are only briefly compared with 


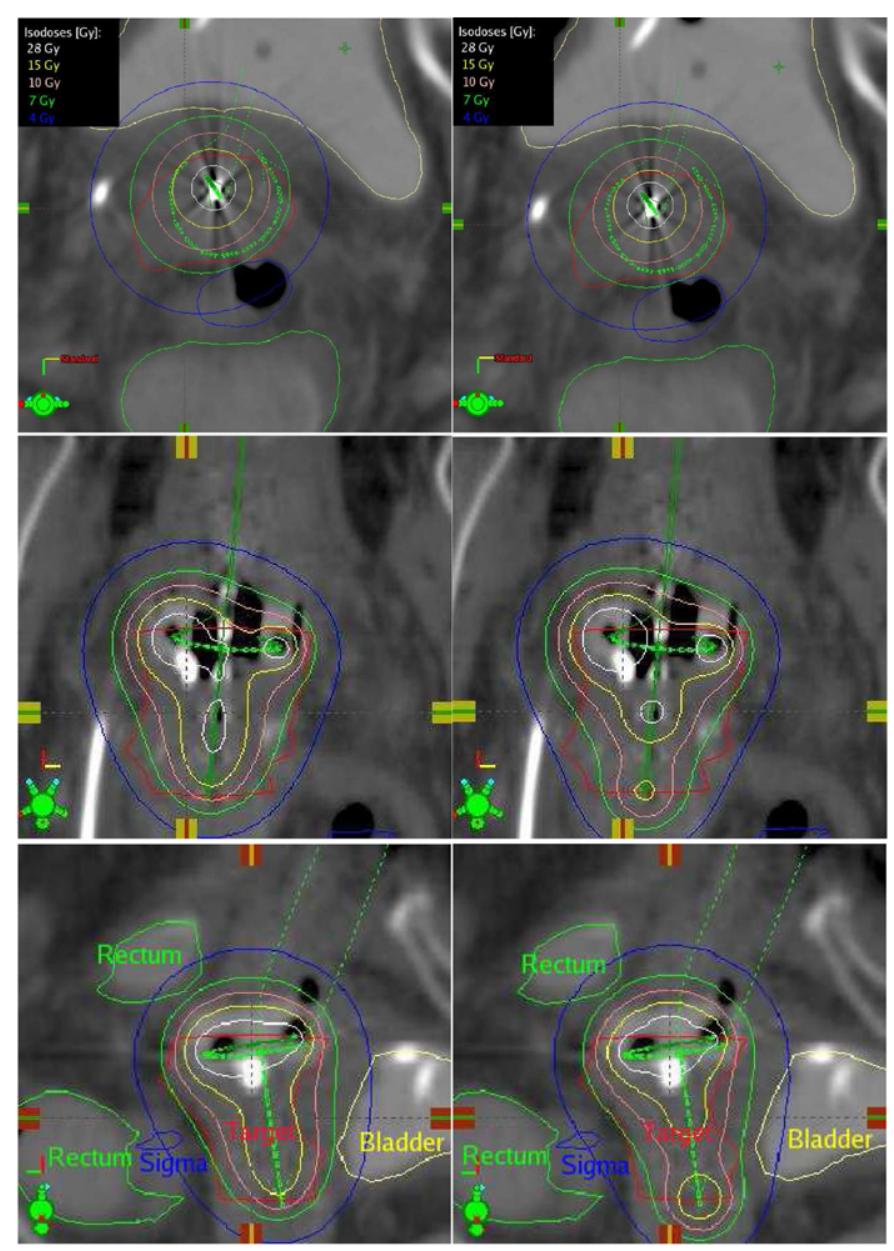

Figure 5. Example 2: left, the final dose distribution with IMOO from different points of view ( $x$-, $y$-, and $z$-directions), and right, for comparison, solution obtained with BrachyVision ${ }^{\circledR}$ optimization. Isodose of $7 \mathrm{~Gy}$ (prescribed dose in target) is the second outermost isodose curve (green in the electronic version). In the electronic version only, target is red, rectum is green, bladder is yellow and sigmoid is blue.

those obtained with the BrachyVision ${ }^{\circledR}$ optimization (figures 5 and 6). In table 3, PTV $90 \%$ dose volume values, OAR $2 \mathrm{~cm}^{3}$ dose volume values and NT volumes, where $50 \%$ of PTV is exceeded are presented. When comparing these solutions, it can be seen that the treatment plan devised with IMOO is better than the plan obtained with the BrachyVision ${ }^{\circledR}$ optimization. In addition, the treatment plan is intuitive and quick to obtain with IMOO, the PTV coverage is better and doses on rectum and NT are lower in the solution obtained with IMOO. In addition, the dose on sigmoid is equal between the solutions, but bladder gets a somewhat lower dose in BrachyVision ${ }^{\circledR}$ optimization. Moreover, when studying the DVHs (figure 6), one can see that OARs get lower doses in the treatment plan obtained with IMOO than with BrachyVision ${ }^{\circledR}$ optimization. Thus, the IMOO treatment plan is superior to the plan obtained with BrachyVision ${ }^{\circledR}$ optimization (which took again about 30 min to obtain). Further, when comparing the planning process, advantages of using IMOO 


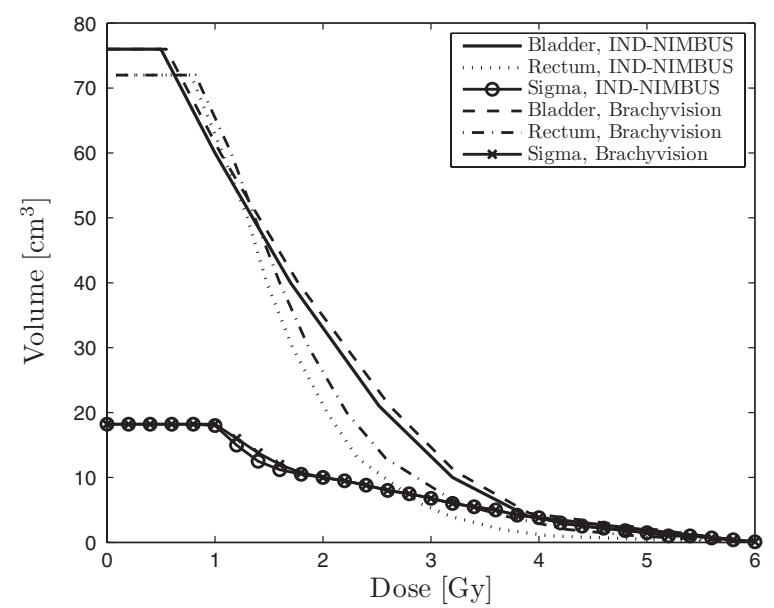

Figure 6. Example 2: for comparison, DVHs of OARs. DVH values start to decrease faster in IMOO solution than in solution obtained with BrachyVision ${ }^{\circledR}$.

Table 3. Example 2: comparison of two solutions with PTV $90 \%$ values, OAR $2 \mathrm{~cm}^{3}$ values and volumes of NT exceeding $50 \%$ of $D_{\text {PTV }}$.

\begin{tabular}{lcc}
\hline & IMOO (Gy) & BrachyVision $^{\circledR}(\mathrm{Gy})$ \\
\hline PTV 90\% & 7.20 & 7.15 \\
Bladder 2 cm & 4.53 & 4.36 \\
Rectum 2 cm & 3.60 & 4.17 \\
Sigmoid 2 cm & 4.71 & 4.71 \\
NT 3.5 Gy & $106.4 \mathrm{~cm}^{3}$ & $122.8 \mathrm{~cm}^{3}$ \\
\hline
\end{tabular}

are obvious: as stated, the treatment planner manipulates directly the desired values of the objective functions, not the objective weights, dwell positions or dwell times, and thus good trade-offs between the solutions could be found and the drawbacks of currently used methods could be overcome.

In the presented examples, there were 17 and 21 continuous decision variables and 508 and 473 sampling points, respectively. The amount of variables can easily be increased in IMOO, but simultaneously the CPU time increases (which affects interactivity). As has been shown, the approach is suitable for instances where the number of variables and sampling points is relatively small (such as in 3D HDR brachytherapy cases). In addition, there were only box constraints for variables. However, it is easy to add any other constraint to our IMOO approach if needed. In addition, the idea of classifying objective functions is intuitive and practical and computation is fast also with different numbers of objective functions, and therefore the number of objectives can be increased. Based on our experiments, it would be reasonable to use convex objective functions which allow the use of a local optimizer. That is because the CPU time increases if a computationally costly global optimizer is needed. Thus, the findings presented in Romeijn et al (2004) are useful. However, the objective functions must represent decision maker's needs because objective values are used as preference information.

One strength of our IMOO approach is that it can easily be applied to any kind of applicators and needles (if temporarily implanted needles are used as applicators). The only input information the system needs is patient geometry and dwell positions. 


\section{Conclusions}

In this paper, we have presented the potential of using the IMOO approach for anatomy-based 3D HDR brachytherapy optimization. In this research, the multiobjective nature of the problem was genuinely taken into account in the problem formulation and in the interactive solution process which was directed by a treatment planner. We have demonstrated the advantages of our interactive approach by two examples of treatment planning for clinical gynecological cancer. Interactive approaches have not been used for brachytherapy before but our examples support their usefulness in this field.

In this study, our IMOO approach was used to determine the dwell time values needed to fulfill the prescribed dose to the tumor and to minimize dose in each organ at risk. In our approach, the treatment planner's knowledge and preferences were used during the iterative optimization process to direct the search in order to find the most preferred treatment plan. This is possible in 3D HDR brachytherapy cases since the computation is fast (number of variables is small) and the treatment planner does not need to wait for the Pareto optimal solution to be generated. IMOO can make treatment planning times shorter and improve the treatment plan's quality because it is capable of handling multiple and strongly conflicting objectives in a convenient way, and thus, it offers a possibility of navigating among the obtained Pareto optimal solutions (i.e. different treatment plans). As seen, there is not much space for improvement of the final treatment plan compared to the other methods but the strength of our approach is its superior interactivity during the planning process. Thus, the drawbacks (e.g. defining weights and trial-and-error planning) of currently used methods can be avoided.

Finally, let us add that this approach brings the planning process near the real clinical issues: with an IMOO system, the treatment planner plays directly with the compromises between a target coverage and protection of OARs instead of manipulating dwell positions, dwell times and objective weights. Whenever a trial-and-error method is used, there are no guarantees for the Pareto optimality of the final solution. In contrast to this, our approach avoids this shortcoming.

\section{Acknowledgments}

The authors wish to thank Joakim Pyyry, MSc(Tech), from Varian Medical Systems Finland, Vesa Ojalehto, MSc, from University of Jyväskylä and Professor Jari Hämäläinen from the University of Eastern Finland. This work was financially supported by TEKES, the Finnish Funding Agency for Technology and Innovation (MASI Technology Programme).

\section{References}

Alterovitz R, Lessard E, Pouliot J, Hsu I, O’Brien J F and Goldberg K 2006 Optimization of HDR brachytherapy dose distributions using linear programming with penalty costs Med. Phys. 33 4012-9

Chajon E, Dumas I, Touleimat M, Magné N, Coulot J, Verstraet R, Lefkopoulos D and Haie-Meder C 2007 Inverse planning approach for 3-D MRI-based pulse-dose rate intracavitary brachytherapy in cervix cancer Int. J. Radiat. Oncol. Biol. Phys. $69955-61$

Craft D, Halabi T and Bortfeld T 2005 Exploration of tradeoffs in intensity-modulated radiotherapy Phys. Med. Biol. 50 5857-68

Craft D, Halabi T, Shih H A and Bortfeld T 2007 An approach for practical multiobjective IMRT treatment planning Int. J. Radiat. Oncol. Biol. Phys. 69 1600-7

Craft D and Bortfeld T 2008 How many plans are needed in an IMRT multi-objective plan database? Phys. Med. Biol. 53 2785-96

Deasy J O 1997 Multiple local minima in radiotherapy optimization problems with dose-volume constraints $M e d$. Phys. 24 1157-61 
DeWitt K D, Hsu I, Speight J, Weinberg V K, Lessard E and Pouliot J 2005 3D inverse treatment planning for the tandem and ovoid applicator in cervical cancer Int. J. Radiat. Oncol. Biol. Phys. 63 1270-4

Ehrgott M and Winz I 2008 Interactive decision support in radiation therapy treatment planning OR Spectr. 30 311-29

Halabi T, Craft D and Bortfeld T 2006 Dose-volume objectives in multi-criteria optimization Phys. Med. Biol. 51 3809-18

Haie-Meder C et al 2005 Recommendations from gynaecological (GYN) GEC-ESTRO working group (I): concepts and terms in 3D image based 3D treatment planning in cervix cancer brachytherapy with emphasis on MRI assessment of GTV and CTV Radiother. Oncol. 74 235-45

Hoffmann A L, Siem A Y D, den Hertog D, Kaanders J H A M and Huizenga H 2006 Derivate-free generation and interpolation of convex Pareto optimal IMRT plans Phys. Med. Biol. 51 6349-69

Hsu I, Lessard E, Weinberg V and Pouliot J 2004 Comparison of inverse planning simulated annealing and geometrical optimization for prostate high-dose-rate brachytherapy Brachytherapy 3 147-52

Küfer K-H, Scherrer A, Monz M, Alonso F, Trinkaus H, Bortfeld T and Thieke C 2003 Intensity-modulated radiotherapy-a large scale multi-criteria programming problem OR Spectr. 25 223-49

Küfer K-H, Monz M, Scherrer A, Süss P, Alonso F, Sultan A S A, Bortfeld Th, Craft D and Thieke Chr 2005 Multicriteria optimization in intensity modulated radiotherapy planning Berichte des Fraunhofer ITWM 77

Lahanas M and Baltas D 2003 Are dose calculations during dose optimization in brachytherapy necessary? Med. Phys. 30 2368-75

Lahanas M, Baltas D and Giannouli S 2003a Global convergence analysis of fast multiobjective gradient based dose optimization algorithms for high dose rate brachytherapy Phys. Med. Biol. 48 599-617

Lahanas M, Baltas D and Zamboglou N 2003b A hybrid evolutionary algorithm for multiobjective anatomy based dose optimization in HDR brachytherapy Phys. Med. Biol. 48 399-415

Lahanas M, Baltas D and Zamboglou N 1999 Anatomy-based three-dimensional dose optimization in brachytherapy using multiobjective genetic algorithms Med. Phys. 26 1904-18

Lahanas M, Milickovic N, Papagiannopoulou M, Baltas D, Zamboglou N and Karouzakis K 2001 Application of a hybrid version of NSGA II for multiobjective dose optimization in brachytherapy Evolutionary Methods for Design, Optimization and Control with Applications to Industrial Problems ed K C Giannakoglou, D T Tsahalis, J Périaux, K D Papailiou and T Fogarty (Athens: International Center for Numerical Methods in Engineering(CIMNE)) pp 299-304

Larichev O 1992 Cognitive validity in design of decision aiding techniques J. Multi-Criteria Decis. Anal. 1 127-38

Lessard E, Hsu I, Aubry J and Pouliot J 2006 SU-FF-T-337: multiobjective inverse planning optimization: adjustment of dose homogeneity and urethra protection in HDR-brachytherapy of the prostate Med. Phys. 332124

Lessard E, Hsu I and Pouliot J 2002 Inverse planning for interstitial gynecologic template brachytherapy: truly anatomy-based planning Int. J. Radiat. Oncol. Biol. Phys. 54 1243-51

Lessard E and Pouliot J 2001 Inverse planning anatomy-based dose optimization for HDR-brachytherapy of the prostate using fast simulated annealing algorithm and dedicated objective function Med. Phys. 28 773-9

Meyer J, Phillips M H, Cho P S, Kalet I and Doctor J N 2004 Application of influence diagrams to prostate intensity-modulated radiation therapy plan selection Phys. Med. Biol. 49 1637-53

Miettinen K 1999 Nonlinear Multiobjective Optimization (Boston, MA: Kluwer)

Miettinen K 2006 IND-NIMBUS for demanding interactive multiobjective optimization Multiple Criteria Decision Making '05 ed T Trzaskalik (Katowice: The Karol Adamiecki University of Economics) pp 137-50

Miettinen K and Mäkelä M M 1995 Interactive bundle-based method for nondifferentiable multiobjective optimization: NIMBUS Optimization 34 231-46

Miettinen K and Mäkelä M M 2002 On scalarizing functions in multiobjective optimization OR Spectr. 24 193-213

Miettinen K and Mäkelä M M 2006 Synchronous approach in interactive multiobjective optimization Eur. J. Oper. Res. 170 909-22

Milickovic N, Lahanas M, Papagiannopoulou M, Baltas D, Zamboglou N and Karouzakis K 2001 Application of multiobjective genetic algorithms in anatomy based dose optimization in brachytherapy and its comparison with deterministic algorithms Evolutionary Methods for Design, Optimization and Control with Applications to Industrial Problems ed K C Giannakoglou, D T Tsahalis, J Périaux, K D Papailiou and T Fogarty (Athens: International Center for Numerical Methods in Engineering (CIMNE)) pp 293-8

Milickovic N, Lahanas M, Papagiannopoulou M, Zamboglou N and Baltas D 2002 Multiobjective anatomy-based dose optimization for HDR-brachytherapy with constraint free deterministic algorithms Phys. Med. Biol. 47 2263-80

Monz M, Küfer K H, Bortfeld T R and Thieke C 2008 Pareto navigation - algorithmic foundation of interactive multi-criteria IMRT planning Phys. Med. Biol. 53 985-98

Morton G C, Sankreacha R, Halina P and Loblaw A 2008 A comparison of anatomy-based inverse planning with simulated annealing and graphical optimization for high-dose-rate prostate brachytherapy Brachytherapy 7 12-6 
Nath R, Anderson L L, Luxton G, Weaver K A, Williamson J F and Meigooni A S 1995 Dosimetry of interstitial brachytherapy sources: recommendations of the AAPM Radiation Therapy Committee Task Group No. 43 Med. Phys. 22 209-34

Pötter R et al 2006 Recommendations from gynaecological (GYN) GEC ESTRO working group (II): concepts and terms in 3D image-based treatment planning in cervix cancer brachytherapy-3D dose volume parameters and aspects of 3D image-based anatomy, radiation physics, radiobiology Radiother. Oncol. 78 67-77

Pouliot J, Kim Y, Lessard E, Hsu I, Vigneron D B and Kurhanewicz J 2004 Inverse planning for HDR prostate brachytherapy used to boost dominant intraprostatic lesion defined by magnetic resonance spectroscopy imaging Int. J. Radiat. Oncol. Biol. Phys. 59 1196-207

Rivard M J, Coursey B M, DeWerd L A, Hanson W F, Saiful Huq M, Ibbott G S, Mitch M G, Nath R and Williamson J F 2004 Update of AAPM Task Group No. 43 report: a revised AAPM protocol for brachytherapy dose calculations Med. Phys. 31 633-74

Romeijn H E, Ahuja R K, Dempsey J F, Kumar A and Li J G 2003 A novel linear programming approach to fluence map optimization for intensity modulated radiation therapy treatment planning Phys. Med. Biol. 48 3521-42

Romeijn H E, Dempsey J F and Li J G 2004 A unifying framework for multi-criteria fluence map optimization models Phys. Med. Biol. 49 1991-2013

Ruotsalainen H, Boman E, Miettinen K and Tervo J 2009 Interactive multiobjective optimization in radiotherapy treatment planning with the Boltzmann transport equation Contemp. Eng. Sci. 2 391-422

Sumida I, Shiomi H, Yoshioka Y, Inoue T, Lessard E, Hsu I and Pouliot J 2006 Optimization of dose distribution for HDR brachytherapy of the prostate using attraction-repulsion model Int. J. Radiat. Oncol. Biol. Phys. 64 643-9

Thieke C, Küfer K-H, Monz M, Scherrer A, Alonso F, Oelfke U, Huber P E, Debus J and Bortfeld T 2007 A new concept for interactive radiotherapy planning with multicriteria optimization: first clinical evaluation Radiother. Oncol. 85 292-8

Yasuo Y, Harada H, Murayama S, Kanazawa K, Nishimura T, Kamata M and Fuji H 2005 Evaluation of anatomybased dwell position and inverse optimization in high-dose-rate brachytherapy of prostate cancer: a dosimetric comparison to a conventional cylindrical dwell position, geometric optimization, and dose-point optimization Radiother. Oncol. 75 311-7

Yu Y 1997 Multiobjective decision theory for computational optimization in radiation therapy Med. Phys. 24 1445-54

Yu Y, Zhang J B, Cheng G, Schell M C and Okunieff P 2000 Multi-objective optimization in radiotherapy: applications to stereotactic radiosurgery and prostate brachytherapy Artif. Intell. Med. 19 39-51 